\title{
INTER-LIMB CENTRE OF PRESSURE SYMMETRY DURING GAIT AMONG STROKE SURVIVORS.
}

Amanda E Chisholm M.Sc. ${ }^{1,2}$

Stephen D Perry Ph.D. 1,2,3

William E McIlroy Ph.D. 1,2,4,5

\section{Affiliations:}

1. Toronto Rehabilitation Institute, Toronto, Ontario, CANADA

2. Graduate Department of Rehabilitation Science, University of Toronto, Toronto, Ontario, CANADA

3. Department of Kinesiology and Physical Education, Wilfrid Laurier University, Waterloo, Ontario, CANADA

4. Department of Kinesiology, University of Waterloo, Waterloo, Ontario, CANADA

5. Sunnybrook Health Science Centre, Toronto, Ontario, CANADA

\section{Acknowledgements:}

The authors acknowledge the support of the Toronto Rehabilitation Institute which receives funding under the Provincial Rehabilitation Research Program from the Ministry of Health and Long-Term Care in Ontario. Support was also provided by Natural Sciences and Engineering Research Council (CGS-M), Canadian Institute of Health Research (MOP-77772) and University of Toronto.

\section{Correspondence:}

Stephen Perry, Ph.D.

Department of Kinesiology \& Physical Education

Wilfrid Laurier University

75 University Avenue West

Waterloo, Ontario, Canada, N2L 3C5

Telephone: 519-884-0710, ext. 4215

Fax: 519-747-4594

sperry@wlu.ca 


\section{INTER-LIMB CENTRE OF PRESSURE SYMMETRY DURING GAIT AMONG STROKE SURVIVORS.}

The purpose of the present study was to describe the spatial-temporal parameters of the centre of pressure (COP) trajectory during the single-support phase of gait among stroke survivors and relate these parameters to the severity of sensorimotor impairment. Fiftyseven participants were asked to walk at their preferred and fast speed over a pressure sensitive mat. Outcome measures included anterior-posterior (AP) COP displacement, AP-COP velocity, medial-lateral (ML) COP variability and foot region COP time. The results demonstrated an asymmetrical AP-COP displacement in favour of the non-paretic limb for the majority of participants. The inter-limb difference scores for AP-COP displacement and AP-COP velocity were related to severity of sensorimotor impairment

and greater among gait aid users. ML-COP variability was greater under the non-paretic limb, possibly suggesting difficultly with paretic limb swing phase. Reduced or absent forefoot COP time suggests difficulty with forward progression and modified foot function during push-off. The inter-limb difference in COP parameters highlights the asymmetrical nature of post-stroke gait and the challenge of maintaining single limb support. We view this information as potentially important to clinicians as an outcome measure for gait rehabilitation.

Keywords: Centre of Pressure; Gait; Stroke; Motor Control; Dynamic Balance 


\section{Introduction}

Impaired walking function is a common neurological deficit following stroke.

General features include decreased velocity, poor efficiency, reduced muscle coordination and spatial-temporal asymmetry. Underlying factors contributing to these changes include loss of muscle control, weakness and/or spasticity [1]. It has been suggested that gait symmetry is an important determinant of post-stroke walking recovery [2]. Understanding the nature and implications of gait symmetry is important due its potential long-term impact on health, such as reduced activity levels and increased risk of musculoskeletal injury [3,4]. Previous research has described gait asymmetry with respect to kinematics, kinetics and spatial-temporal measures [1,2,4-7]. Chen et al. reported several gait differences, such as swing time asymmetry, between stroke survivors and control participants at matched speeds, the results suggest impaired paretic limb swing initiation and single stance [8]. The ability to maintain single limb support has previously been identified as an important determinant of gait stability and current indices of temporal symmetry may partially reflect this challenge $[9,10]$.

The centre of pressure (COP) represents the cumulative neuromuscular response that controls the centre of mass (COM) movement to help maintain forward progression and upright balance. The anterior-posterior (AP) COP trajectory may indicate the control of the COM forward progression during the stance phase. Conversely, medial-lateral (ML) COP movement may largely reflect the control process to regulate lateral stability, especially in the single-support phase, and the ability to shift weight between limbs. Additionally, the COP trajectory may change as gait velocity increases due to reduced 
single-support time, thus possibly providing insight on specific mechanisms of gait dysfunction.

One previous study has examined inter-limb differences in the COP trajectory during gait initiation after stroke. A subgroup of stroke survivors with equinusvarus foot demonstrated an initial COP displacement posteriorly under the paretic limb and anteriorly under the non-paretic limb [5]. The work highlighted the asymmetrical nature of post-stroke gait. While most studies evaluating gait have restricted their focus to spatial-temporal or kinetic asymmetry $[4,6,8]$, the analysis of stance phase COP excursions may reveal additional information regarding underlying control challenges.

The current study focuses on potential inter-limb differences in spatial-temporal parameters of the COP trajectory throughout the single-support phase among individuals with stroke. We hypothesized that paretic limb COP trajectory compared to the nonparetic limb, would demonstrate a (1) shorter AP-COP displacement (2) slower AP-COP velocity (3) greater ML-COP variability and (4) higher percentage of single-support time under the mid-foot (as compared to heel and forefoot). Additionally, we propose that increasing walking velocity, greater than preferred, will exacerbate the limb differences, specifically revealing an increase in ML-COP variability. The second objective was to examine the relationships between inter-limb COP parameters and (1) severity of lower extremity sensorimotor impairment, and (2) single-support time symmetry. It was hypothesized that individuals with greater lower extremity sensorimotor impairment and single-support time asymmetry would demonstrate larger inter-limb COP differences. 


\section{Methods}

Participants

Fifty-seven individuals who sustained a stroke (hemorrhagic or ischemic) and were able to walk $10 \mathrm{~m}$ independently with or without an assistive device participated. This study was approved by local university and hospital research ethics review boards. All participants provided informed consent.

Protocol

Participants were asked to walk a distance of $7 \mathrm{~m}$ over a pressure sensitive mat under two conditions: preferred speed and fastest speed at which they felt safe. Each walking trial started $1 \mathrm{~m}$ before the mat and finished $1 \mathrm{~m}$ after the mat. Three trials were performed for each condition; rest was permitted as needed. Participants wore their regular footwear and were encouraged to walk without gait aids (walker/cane and anklefoot orthotic) if possible.

Measurements

The Chedoke-McMaster Stroke Assessment (CMSA) Impairment Inventory was performed to assess severity of sensorimotor impairment of the leg and foot by a physiotherapist or trained research assistant. Each item was measured on a 7-point scale with lower scores indicating greater impairment. The CMSA has a high inter-rater and intra-rater reliability [11].

All gait measurements were recorded using a pressure sensitive mat (GAITRite ${ }^{\circledR}$ System, CIR Systems, Clifton, NJ), which was $5.25 \mathrm{~m}$ long and $0.88 \mathrm{~m}$ wide. Pressure sensors were scanned at a frequency of $80 \mathrm{~Hz}$ to record the location, time and pressure level of active sensors. The GAITRite system identifies the maximum vertical pressure 
level and expresses each pressure value as a percentage of the maximum and then divides them into 7 levels [12]. The time point and geometry of the active sensors allow for the calculation of spatial-temporal parameters.

\section{Data Analysis}

Data analysis software included with the GAITRite ${ }^{\circledR}$ system automatically outputs spatial-temporal parameters for each trial, mean and standard deviation (SD) scores were calculated for each condition. All symmetry measures were calculated as paretic/non-paretic. The COP was calculated from the raw pressure time series data extracted from the GAITRaw ${ }^{\circledR}$ software. A custom software program was designed to analyze spatial-temporal parameters of the COP trajectory.

Four consecutive foot contacts (two paretic and two non-paretic) recorded from the middle of the mat were analyzed per trial. In the custom software program, the lateral border of each foot contact was identified and the foot width to create a parallelogram. Only foot contacts with an identifiable heel, mid-foot and forefoot region (>70\% of actual foot length) were used in the analysis (excluded 5 trials). The foot contact was divided into three equal sections along the length to identify heel, mid-foot and forefoot regions (Figure1). COP points occurring within the parallelogram at a time when no pressure was recorded outside of the parallelogram were identified as the single-support phase. COP analysis was based only on the single-support phase.

The primary outcome measures included the AP-COP displacement, average APCOP velocity, ML-COP variability and COP time within the each foot region. All COP parameters measured are relative to the foot's lateral border. Foot angle was measured between the foot's lateral border and the line of progression for each limb per condition. 
AP-COP displacement was defined as the AP distance between the initial and final COP points identified during the single support phase; expressed as a percentage relative to foot length. AP-COP velocity was calculated as the rate of change in AP position with respect to time; expressed as an average over the total single-support phase and within each foot region. ML-COP variability was measured as the SD of the medial-lateral COP position relative to the foot's lateral border. COP time was defined as the amount of time the COP spent in each foot region and expressed as a percentage of the total singlesupport duration. Each COP parameter was corrected for foot angle.

A three-factor, one between subject factor (group/aid) and two within subject factors (limb; gait speed), repeated measures ANOVA compared COP parameters. There were 2 levels for each of the 3 factors: 1) aid (gait aid vs. no gait aid), 2) limb (paretic vs. non-paretic) and 3) gait speed (preferred vs. fast). Average single-support time for each limb per trial was used as a co-variate in the ANOVA. Post-hoc Tukey analysis was conducted on pair-wise comparisons. Outliers were identified as three standard deviations (SD) from the participant's mean score and removed after justification from visual inspection ( $\mathrm{n}=12$ foot contacts). Forefoot region data (velocity and time) was not normally distributed as determined by scores $<0.95$ on the Shapiro-Wilk test and data was ranked transformed prior to statistical analysis. Spearman's non-parametric ranked correlation was performed to relate CMSA leg and foot scores to the average ratio (paretic/non-paretic) for COP parameters during the preferred condition $(\mathrm{df}=55)$. Singlesupport time symmetry was related to the average ratio (paretic/non-paretic) for COP parameters in the preferred condition with a Pearson's correlation $(\mathrm{df}=55)$. Reported values are mean \pm SD. Statistical significance was set at $\mathrm{p}<0.05$. 


\section{Results}

\section{Characteristics of COP trajectory}

Participant demographic and clinical data are outlined in Table 1. Comparisons between limbs, groups and speeds for COP measures are highlighted in Table 2. The effect of foot angle did not change the results comparing group, limb and condition; therefore COP values reported are relative to the foot's lateral border. The non-paretic limb AP-COP displacement was significantly longer than the paretic limb during the preferred and fast conditions ( $\mathrm{p}=0.004$; Figure2). For the main effect of group/aid, gait aid (GA) users displayed a shorter AP-COP displacement as compared to the non gait aid (NGA) users ( $\mathrm{p}=0.013)$. A significant interaction between group and limb revealed a larger inter-limb difference for the GA group $(\mathrm{p}=0.019)$. Single-support time was positively related to AP-COP displacement ( $\mathrm{p}=0.021)$. Most participants $(43 / 57)$ demonstrated a longer non-paretic AP-COP displacement. The mean AP-COP displacement inter-limb difference was $12.8 \%$ for participants with a greater non-paretic AP-COP displacement $(n=43)$ compared to $5.1 \%$ for participants with a greater paretic AP-COP displacement ( $n=14)$. AP-COP displacement was significantly increased during the fast condition by $5.9 \%$ and $5.6 \%$ under the paretic and non-paretic limbs, respectively $(\mathrm{p}<0.001)$.

There was a trend towards a greater average AP-COP velocity under the nonparetic vs. paretic limb ( $\mathrm{p}=0.059)$. The GA group had a slower average AP-COP velocity $(\mathrm{p}=0.004)$ and greater difference between limbs $(\mathrm{p}=0.008)$ compared to the NGA group. The main effect of gait speed (preferred vs. fast) revealed a significant difference in 
average AP-COP velocity with an increase of $7.5 \mathrm{~cm} / \mathrm{s}(\mathrm{p}<0.001)$. Single-support time was negatively related to average AP-COP velocity $(\mathrm{p}=0.01)$.

Foot region analysis revealed comparable mean AP-COP velocities between limbs in the heel, mid-foot and forefoot ( $\mathrm{p}>0.05)$. The GA group had a slower AP-COP velocity in all foot regions compared to the NGA group $(\mathrm{p}<0.05)$. The group and limb interaction was significant in the mid-foot $(\mathrm{p}=0.024)$; GA group demonstrated a larger inter-limb difference compared to the NGA group. Single-support time was not related to mean AP-COP velocity in all foot regions ( $>>0.05$ ). In the fast condition, average APCOP velocity increased by $4.5 \mathrm{~cm} / \mathrm{s}, 12.3 \mathrm{~cm} / \mathrm{s}$ and $8.7 \mathrm{~cm} / \mathrm{s}$ for the heel, mid-foot and forefoot regions, respectively $(\mathrm{p}<0.001)$. A significant interaction between speed and limb occurred in the forefoot, wherein velocity increased more for the non-paretic limb between preferred and fast conditions compared to the paretic limb $(\mathrm{p}=0.001)$.

ML-COP SD revealed a main effect for limb, with higher scores under the nonparetic limb ( $\mathrm{p}=0.001$, Figure2). ML-COP position scores were not significantly different between limbs $(\mathrm{p}=0.238)$. There was no difference between groups for MLCOP position and SD $(\mathrm{p}>0.05)$. Fast walking had a ML-COP position $(\mathrm{p}<0.001)$ closer to the foot's medial border with higher SD ( $\mathrm{p}=0.041)$ scores compared to the preferred condition. Single-support time was positively related to position and negatively related to ML-COP SD $(\mathrm{p}<0.05)$.

The percentage of single-support COP time under the mid-foot region was comparable between limbs $(\mathrm{p}=0.561)$. No difference was found between limbs for COP time spent in the heel $(\mathrm{p}=0.755)$, while there was a trend toward more time on the nonparetic vs. paretic forefoot $(\mathrm{p}=0.053)$. The NGA group spent significantly more time on 
the forefoot compared to the GA group $(\mathrm{p}=0.048)$. In the fast condition, COP spent more time under the forefoot $(\mathrm{p}<0.001)$ with a trend towards less time under the heel ( $p=0.057)$, and no change for the mid-foot $(\mathrm{p}=0.38)$ compared the preferred condition. The interaction between speed and limb was statistically significant in the mid-foot $(\mathrm{p}=0.027)$ and forefoot $(\mathrm{p}=0.001)$. Single-support time was not significant related to COP time spent in any foot region ( $\mathrm{p}>0.05$ ). Only 30/57 participants had COP excursion into the defined forefoot region during the single-support phase for both limbs. While, 23/57 only used the forefoot region of one limb and 4/57 did not have a forefoot COP excursion in either limb.

Correlations between COP trajectory, impairment and single-support time

The AP-COP displacement ratio (paretic/non-paretic) displayed a significant positive relationship to CMSA foot $(\mathrm{r}=0.521)$ and leg $(\mathrm{r}=0.485)$ scores $(\mathrm{p}<0.05$; Figure3). Similarly, CMSA foot $(r=0.415, r=0.529, \mathrm{p}<0.05)$ and $\operatorname{leg}(r=0.340, r=0.51, p<0.05)$ scores were significantly related to AP-COP velocity ratio and ML-COP SD ratio (Figure3), respectively. The mid-foot region time ratio did not reveal a significant correlation to CMSA foot $(\mathrm{r}=-0.145)$ and leg $(\mathrm{r}=-0.069)$ scores $(\mathrm{p}>0.05)$.

Pearson’s correlation revealed a significant relationship between single-support time symmetry and inter-limb AP-COP displacement ( $\mathrm{r}=0.756, \mathrm{p}<0.001)$, ML-COP SD $(\mathrm{r}=0.598, \mathrm{p}<0.001)$ and overall AP-COP velocity ratios $(\mathrm{r}=0.551, \mathrm{p}<0.001)$. Similarly, AP-COP velocity ratio in the mid-foot $(r=0.355, \mathrm{p}=0.007)$ and forefoot $(r=0.333$, $\mathrm{p}=0.041, \mathrm{df}=36)$ displayed a significant correlation, while heel did not $(\mathrm{r}=-0.111$, $\mathrm{p}=0.412$ ). ML-COP position was not significantly correlated with single-support time symmetry $(r=0.083, p=0.54)$. Single-support time symmetry and COP time ratio was 
significantly related in the forefoot $(r=0.325, \mathrm{p}=0.047)$, but not the heel or mid-foot ( $\mathrm{r}=-$ $0.183, \mathrm{r}=0.182, \mathrm{p}>0.05)$.

\section{Discussion}

We compared the COP trajectory under the paretic and non-paretic limbs during the single support phase of gait. On average, the results illustrate an asymmetric COP trajectory in regards to AP displacement and ML variability. In contrast, no differences were found between limbs for average AP-COP velocity, and mid-foot region COP time. Gait aid users had greater inter-limb AP-COP asymmetry for displacement and velocity. Fast walking increased inter-limb asymmetry for AP-COP velocity and forefoot COP time. Participants with asymmetric COP excursions were likely to exhibit greater lower extremity sensorimotor impairment and single-support time asymmetry. Evidence of asymmetry

Our results are supported by previous work reporting temporal gait asymmetry among stroke survivors [1,2,4-8]. The observed asymmetrical AP-COP displacement between limbs is corroborated by Rodgers et al, who reported 0.81 ratio among chronic stroke survivors ( $>6$ months post-stroke) without using gait aids [13]. Our participants (1-134 months post-stroke), demonstrated an average inter-limb AP-COP displacement ratio of 0.65 and 0.84 for gait aid users and non-users, respectively. AP displacement asymmetry is complemented by the tendency for reduced COP time and slower AP velocity on the paretic vs. non-paretic forefoot. Approximately $40 \%$ of participants had absent COP excursions in either paretic or non-paretic forefoot. Additionally, increased ML-COP variability under the non-paretic vs. paretic limb is consistent with a larger ML- 
COP range previously reported [13]. This behaviour may be an adaptive process to compensate for impaired sensorimotor control of the paretic limb during gait.

\section{Sources of inter-limb COP asymmetry}

Possible reasons for asymmetry may be found in associations between inter-limb COP ratio and lower extremity sensorimotor impairment. Individuals with greater sensorimotor impairments were likely to exhibit more asymmetric COP excursions. This finding corresponds with previous studies using other motor impairment measures with different symmetry indices [4,7]. Specifically, AP-COP displacement asymmetry may be related to challenges in controlling forward progression during single-support over the paretic limb. Previously observed changes in plantar dynamics coincide with asymmetrical COP excursions, such as delayed non-paretic heel-off and toe-off may create a greater mid-stance interval and a flat foot initial contact on the paretic side [9]. Ankle plantarflexors spasticity can produce a flat foot initial contact and has been indicated as an important determinant of single-support time asymmetry at preferred and fast walking [14]. Participants who displayed a shorter non-paretic AP-COP displacement may reveal a different underlying control challenge. For example, inadequate propulsion swinging paretic limb due to ankle plantarflexors and/or hip flexors weakness may result in a shorter paretic step length and reduced COM displacement [8]. Previous studies have reported step length asymmetry among stroke survivors; however it tends to be less prevalent and varies in direction $[4,15]$. Additionally, a few participants were temporally asymmetric towards the paretic limb, which may contribute to greater paretic AP-COP displacement. 
Participants with severe impairment were likely to demonstrate a greater MLCOP variability asymmetry. This finding parallels the association between ML-COP variability and single-support time asymmetry. Increased time on the non-paretic limb to compensate for the paretic limb may facilitate larger ML-COP variability. Compensatory movements observed during paretic swing phase, such as hip hiking and lower limb circumduction, may also increase ML-COP variability in those with greater lower limb impairment [8].

\section{Influence of gait aids on inter-limb COP asymmetry}

The magnitude of inter-limb AP-COP asymmetry appears to be greater among gait aids users, despite similar single-support time asymmetry between groups. These devices may help participants reduce loading the paretic limb during stance and allow the upper extremity to assist with balance control. Gait aid users exhibited greater lower limb impairment at the knee and hip joints compared to non-users, which may contribute to their asymmetric gait pattern. A shorter AP-COP displacement and slower AP-COP velocity was observed among gait aid users vs. non-users; however these results may simply reflect their slower walking velocity. Previous studies have implicated that gait aids may alter the degree and pattern of inter-limb symmetry [15]. Among sub-acute stroke survivors, using a cane improved temporal symmetry only in patients who demonstrated asymmetric gait when walking without any assistive device [16]. Similarly, another study reported that gait aids had little influence on spatial-temporal symmetry, except for participants with severe hemiplegia who exhibited improved swing time symmetry [17]. The influence of gait aids remains unclear and future work should 
examine whether gait aids improve or exacerbate inter-limb COP symmetry among individuals who require them for functional mobility.

Influence of speed on inter-limb COP asymmetry

In response to fast walking, single-support AP-COP displacement and velocity increased for both limbs with no change in symmetry. However, the non-paretic limb increased forefoot AP-COP velocity and COP time more than the paretic limb. It is possible that the paretic limb may have difficulty generating propulsive forces required for fast walking due to ankle muscle weakness and/or spasticity [1,7]. Our findings show that within participants ML-COP variability increased during fast walking. Previous work on chronic stroke survivors reported higher ML-COP variability among individuals with a faster preferred gait velocity [18]. Greater ML variability may reflect an increased demand on lateral stability when challenged to walk faster. The effects of gait velocity on inter-limb COP symmetry may depend on the specific underlying impairments and the strategy used to increase walking speed.

\section{Conclusion}

In conclusion, the non-paretic limb COP excursions demonstrated a greater AP displacement, AP velocity, ML variability and forefoot time during single support compared to the paretic limb. Stroke survivors using gait aids displayed greater interlimb COP asymmetry for AP displacement and AP velocity. As well, inter-limb asymmetry was more evident for individuals with severe lower extremity sensorimotor control impairment. Altered COP parameters may represent difficulties in controlling balance and forward progression over the paretic limb. Continued study needs to examine within subject effects of assistive devices and gait speed. Overall this work 
highlights specific post-stroke gait characteristics and presents a novel approach to measuring COP trajectories during gait.

\section{References}

1. Olney SJ and Richards CL, Hemiparetic gait following stroke. Part I: Characteristics. Gait Posture 1996;4:136-148.

2. Hodt-Billington C, Helbostad JL and Moe-Nilssen R, Should trunk movement or footfall parameters quantify gait asymmetry in chronic stroke patients? Gait Posture 2008;27:552-558.

3. Michael KM, Allen JK and Macko RF, Reduced ambulatory activity after stroke: The role of balance, gait, and cardiovascular fitness. Arch Phys Med Rehabil 2005;86:15521556.

4. Patterson KK, Parafianowicz I, Danells CJ, et al. Gait asymmetry in communityambulating stroke survivors. Arch Phys Med Rehabil 2008;89:304-310.

5. Bensoussan L, Mesure S, Viton JM and Delarque A, Kinematic and kinetic asymmetries in hemiplegic patients' gait initiation patterns. J Rehabil Med 2006;38:287294.

6. Kim CM, Eng JJ. Magnitude and pattern of 3D kinematic and kinetic gait profiles in persons with stroke: Relationship to walking speed. Gait Posture 2004;20:140-146.

7. Bowden MG, Balasubramanian CK, Neptune RR and Kautz SA, Anterior-posterior ground reaction forces as a measure of paretic leg contribution in hemiparetic walking. Stroke. 2006;37:872-876.

8. Chen G, Patten C, Kothari DH and Zajac FE, Gait differences between individuals with post-stroke hemiparesis and non-disabled controls at matched speeds. Gait Posture 2005;22:51-56.

9. Gaviria M, D'Angeli M, Chavet P, Pelissier J, Peruchon E and Rabischong P, Plantar dynamics of hemiplegic gait: a methodological approach. Gait Posture 1996;4:297-305

10. Perry J, The mechanics of walking in hemiplegia. Clin.Orthop.Relat.Res 1969;63:2331

11. Gowland C, Stratford P, Ward M, Mo.reland J, Torresin W, Van Hullenaar S, Sanford J, Barreca S, Vanspall B and Plews N, Measuring physical impairment and disability with the Chedoke-McMaster Stroke Assessment. Stroke 1993;24:58-63. 
12. GaitRite Systems - The GAITRite Electronic Walkway Measurements \& Definitions. http://www.gaitrite.com/Downloads/index.html. 2008:1-16.

13. Rodgers M, Forrester L, Mizelle C and Harris-Love ML, Effects of gait velocity on COP symmetry measures in individuals with stroke. Proceedings of the 28th Annual Meeting of the American Society of Biomechanics September 2004.

14. Hsu AL, Tang PF and Jan MH, Analysis of impairments influencing gait velocity and asymmetry of hemiplegic patients after mild to moderate stroke. Arch Phys Med Rehabil 2003;84:1185-93.

15. Kim CM and Eng JJ, Symmetry in vertical ground reaction force is accompanied by symmetry in temporal but not distance variables of gait in persons with stroke. Gait Posture 2003;18:23-28.

16. Beauchamp MK, Skrela M, Southmayd D, Trick J, Van Kessel M, Brunton K, Inness $\mathrm{E}$ and McIlroy WE, Immediate effects of cane use on gait symmetry in individuals with subacute stroke. Physiother Can 2009;61:154-160.

17. Tyson SF, Hemiplegic gait symmetry and walking aids. Physiotherapy Theory and Practice 1994;10:153-159.

18. Mizelle C, Rodgers M and Forrester L, Bilateral foot center of pressure measures predict hemiparetic gait velocity. Gait Posture 2006; 24: 356-363. 
Table 1 - Summary of participant demographics and clinical characteristics

\begin{tabular}{lll}
\hline Characteristic & $\begin{array}{l}\text { Gait Aid Group } \\
(\mathrm{n}=25)\end{array}$ & $\begin{array}{l}\text { No Gait Aid Group } \\
(\mathrm{n}=32)\end{array}$ \\
\hline Male/Female & $15 / 10$ & $22 / 10$ \\
Age & $63.8 \pm 12.5(41-85)$ & $62.2 \pm 11.8(36 .-83)$ \\
Time Post-stroke (months) & $28.8 \pm 28.0(1-92.7)$ & $46.1 \pm 31.5(1-134)^{*}$ \\
Type of Stroke (I/H/U) & $20 / 4 / 1$ & $18 / 5 / 9$ \\
Side of Paresis (L/R) & $11 / 14$ & $15 / 17$ \\
AFO (Y:N) & $8 / 17$ & $2 / 30 *$ \\
CMSA leg score & $4.7 \pm 1.2(3-6)$ & $5.6 \pm 1.1(3-7)^{*}$ \\
CMSA foot score & $4.3 \pm 1.7(2-7)$ & $4.7 \pm 1.8(2-7)$ \\
NIHSS & $4.2 \pm 2.6(0-9)$ & $2.5 \pm 2.5(0-8)^{*}$ \\
Gait - Preferred Condition & & \\
$\quad$ Velocity (cm/s) & $59.6 \pm 23.7(21.8-96.6)$ & $83.0 \pm 32.5(27.8-150.2)^{*}$ \\
$\quad$ Stance time symmetry & $0.92 \pm 0.1(1.07-0.78)$ & $0.93 \pm 0.1(1.06-0.72)$ \\
$\quad$ Swing time symmetry & $1.24 \pm 0.3(1.79-0.83)$ & $0.92 \pm 0.1(1.11-0.56)$ \\
Gait - Fast Condition & & \\
$\quad$ Velocity (cm/s) & $85.7 \pm 33.7(20.9-134.2)$ & $114.1 \pm 45.9(40.6-216.7)^{*}$ \\
$\quad$ Stance time symmetry & $0.91 \pm 0.1(1.02-0.74)$ & $1.23 \pm 0.3(1.97-0.89)$ \\
$\quad$ Swing time symmetry & $1.25 \pm 0.3(1.8-0.95)$ & $1.21 \pm 0.3(1.89-0.89)$ \\
\hline
\end{tabular}

Notes: Values are mean \pm SD (range). Gait aid includes two/four wheel walkers, single and quad point canes. I: Ischemic, H: Hemorrhagic, U: Unknown, L: Left, R: Right. CMSA: Chedoke McMaster Stroke Assessment - evaluates the level of leg and foot impairment. NIHSS: National Institute of Health Stroke Scale - evaluates the degree of neurological deficit. Stance and swing time symmetry $=$ paretic/non-paretic.

* Significant group comparison, $\mathrm{p}<0.05$. 
Table 2 - Summary of Centre of Pressure (COP) Outcome Measures

Mean \pm SD scores and ANOVA results

\begin{tabular}{|c|c|c|c|c|c|c|c|c|}
\hline & \multicolumn{4}{|c|}{ Gait Aid Group } & \multicolumn{4}{|c|}{ No Gait Aid Group } \\
\hline & \multicolumn{2}{|c|}{ Preferred speed } & \multicolumn{2}{|c|}{ Fast speed } & \multicolumn{2}{|c|}{ Preferred speed } & \multicolumn{2}{|c|}{ Fast speed } \\
\hline & Paretic & Non-Paretic & Paretic & Non-Paretic & Paretic & Non-Paretic & Paretic & Non-Paretic \\
\hline Single-Support Time $(\mathrm{s})^{\mathrm{b}, \mathrm{c}, \mathrm{d}}$ & $0.43 \pm 0.07$ & $0.53 \pm 0.13$ & $0.39 \pm 0.06$ & $0.49 \pm 0.12$ & $0.4 \pm 0.04$ & $0.48 \pm 0.11$ & $0.35 \pm 0.04$ & $0.43 \pm 0.11$ \\
\hline AP-COP Displacement (\%) & $18.6 \pm 9.7$ & $30.4 \pm 11.2$ & $25.2 \pm 11.7$ & $36.3 \pm 11.2$ & $30.6 \pm 16.1$ & $36.5 \pm 11.3$ & $36.0 \pm 16.6$ & $42.0 \pm 11.7$ \\
\hline \multicolumn{9}{|l|}{ AP-COP Velocity $(\mathrm{cm} / \mathrm{s})$} \\
\hline Heel $^{b, c}$ & $6.1 \pm 7.8$ & $11.2 \pm 13.5$ & $11.2 \pm 14.4$ & $14.4 \pm 17.1$ & $15.3 \pm 14.4$ & $14.9 \pm 13.1$ & $21.2 \pm 19.6$ & $18.8 \pm 15.3$ \\
\hline Mid-foot ${ }^{b, c, f}$ & $15.9 \pm 10.4$ & $21.3 \pm 10.7$ & $23.3 \pm 14.8$ & $30.0 \pm 16.9$ & $29.8 \pm 21.1$ & $31.6 \pm 21.6$ & $45.8 \pm 36.0$ & $47.5 \pm 35.3$ \\
\hline Forefoot ${ }^{b, c, g}$ & $1.8 \pm 6.8$ & $5.7 \pm 11.1$ & $6.7 \pm 17.0$ & $14.7 \pm 19.4$ & $10.4 \pm 22.2$ & $13.9 \pm 24.8$ & $16.9 \pm 28.7$ & $27.0 \pm 33.8$ \\
\hline Total $a, b, c, f$ & $12.3 \pm 6.0$ & $15.6 \pm 6.6$ & $18.8 \pm 9.3$ & $21.5 \pm 8.5$ & $21.7 \pm 11.3$ & $21.8 \pm 9.2$ & $30.5 \pm 15.2$ & $30.2 \pm 13.2$ \\
\hline \multicolumn{9}{|l|}{$\mathrm{ML}-\mathrm{COP}(\mathrm{cm})$} \\
\hline Position $a, c, f$ & $4.57 \pm 0.99$ & $4.39 \pm 0.87$ & $4.86 \pm 0.92$ & $4.69 \pm 0.79$ & $4.59 \pm 0.85$ & $4.74 \pm 0.86$ & $4.75 \pm 0.81$ & $4.85 \pm 0.89$ \\
\hline $\mathrm{SD}^{\mathrm{a}, \mathrm{c}, \mathrm{d}}$ & $0.27 \pm 0.23$ & $0.61 \pm 0.44$ & $0.33 \pm 0.23$ & $0.69 \pm 0.47$ & $0.39 \pm 0.27$ & $0.60 \pm 0.34$ & $0.51 \pm 0.31$ & $0.72 \pm 0.37$ \\
\hline \multicolumn{9}{|l|}{ COP Time (\%) } \\
\hline Heel & $31.8 \pm 34.2$ & $35.7 \pm 30.3$ & $24.6 \pm 28.5$ & $29.5 \pm 26.7$ & $35.8 \pm 28.1$ & $35.6 \pm 25.5$ & $36.1 \pm 25.0$ & $32.4 \pm 23.4$ \\
\hline Mid-foot ${ }^{\mathrm{g}}$ & $66.9 \pm 33.9$ & $61.3 \pm 29.2$ & $71.5 \pm 27.6$ & $59.9 \pm 25.3$ & $57.9 \pm 28.6$ & $56.8 \pm 24.2$ & $55.1 \pm 27.9$ & $50.5 \pm 24.2$ \\
\hline Forefoot ${ }^{b, c, g}$ & $0.6 \pm 3.8$ & $3.0 \pm 7.4$ & $4.1 \pm 10.3$ & $10.6 \pm 16.7$ & $5.6 \pm 11.5$ & $8.0 \pm 12.4$ & $8.9 \pm 14.6$ & $17.1 \pm 17.5$ \\
\hline
\end{tabular}

ANOVA results were statistically significant at $\mathrm{p}<0.05$ for the following effects;

${ }^{\mathrm{a}}$ Single support time, ${ }^{\mathrm{b}}$ Group, ${ }^{\mathrm{c}}$ Speed, ${ }^{\mathrm{d}}$ Limb, ${ }^{\mathrm{e}}$ Group*Speed, ${ }^{\mathrm{f}}$ Group*Limb, ${ }^{\mathrm{g}}$ Speed*Limb, ${ }^{\mathrm{h}}$ Group*Speed*Limb 
Figure 1

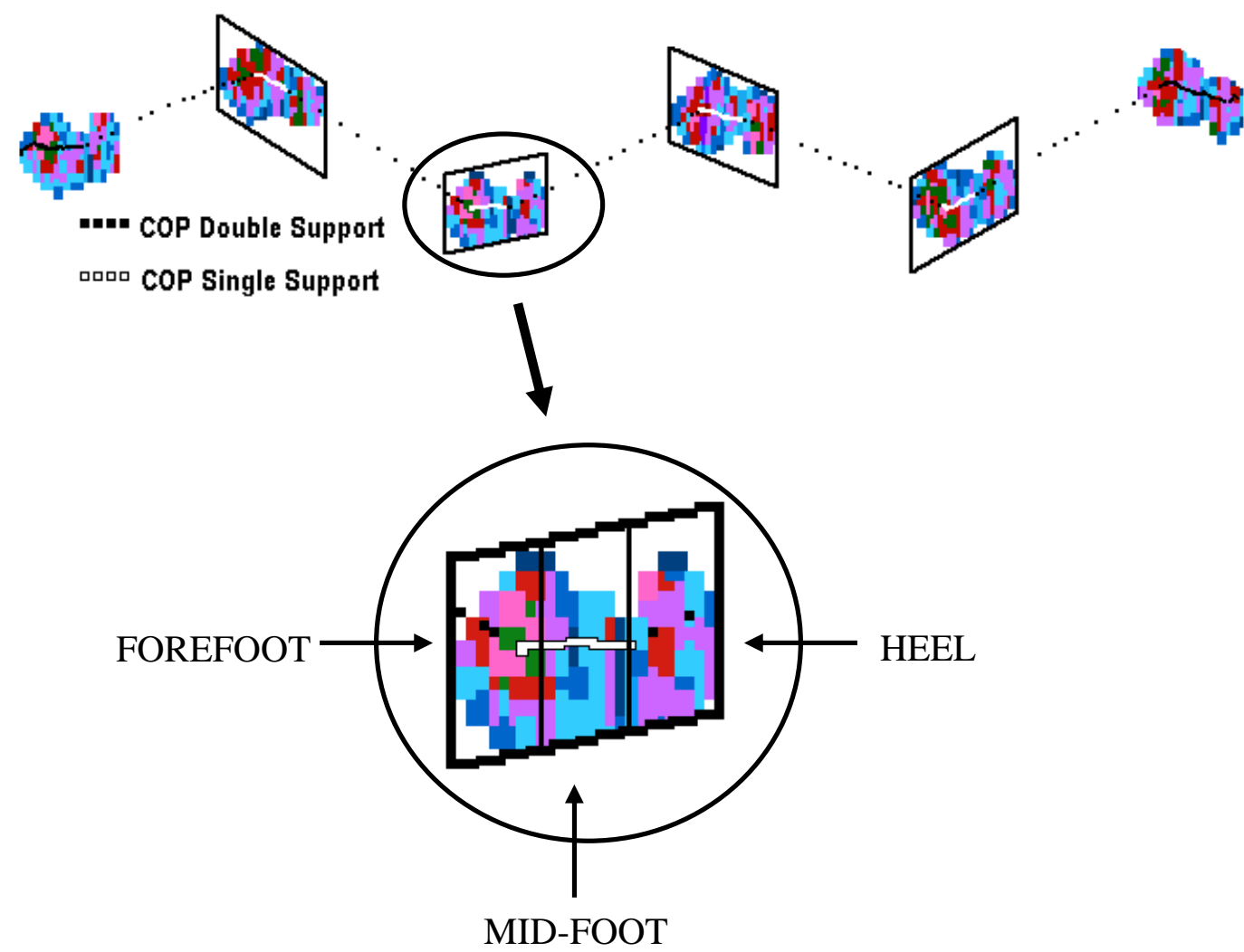

Figure 1: An illustration of the custom software program displaying each foot contact and the graded pressure levels under different foot regions. The four parallelograms that enclose each foot contact are used in the analysis. Centre of pressure (COP) points occurring within the parallelogram at a time when no pressure is recorded outside of the parallelogram is identified as the single support phase. The white COP points indicate the single support phase and black COP points indicate double support. Each parallelogram was divided into three equal sections along the length to identify the heel, mid-foot and forefoot regions. 
Figure 2
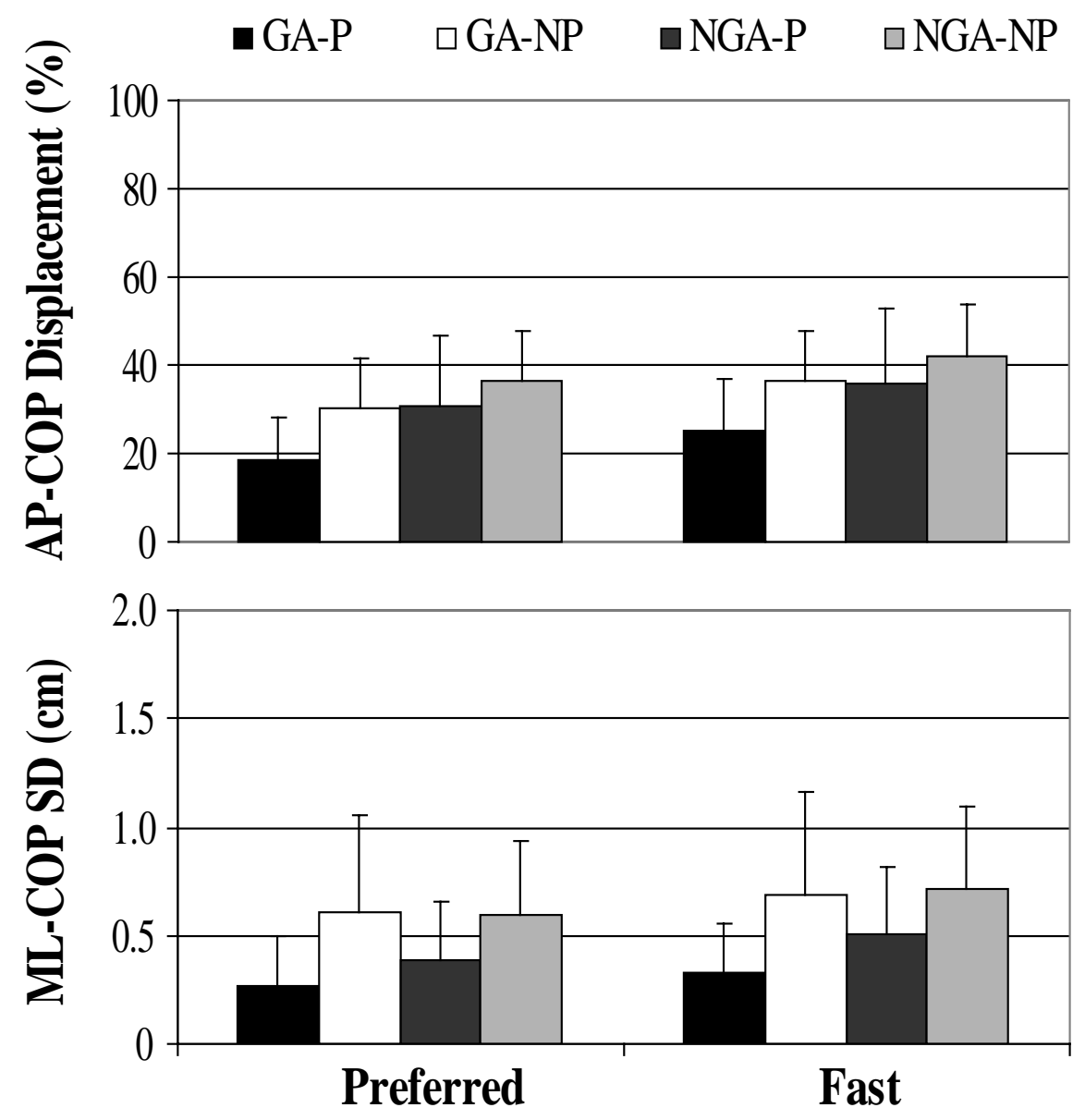

Figure 2: AP-COP displacement and ML-COP SD for the paretic and non-paretic limbs are illustrated per each group and condition. The GA group had a shorter AP-COP displacement and greater inter-limb asymmetry compared to the NGA group $(\mathrm{p}<0.05)$. The non-paretic limb demonstrated greater AP-COP displacements compared to the paretic limb $(\mathrm{p}=0.004)$. Fast walking produced significantly longer AP-COP displacements $(\mathrm{p}<0.001)$. ML-COP SD scores were significantly different between limbs $(\mathrm{p}=0.001)$. Fast walking had higher ML-COP SD scores compared to preferred walking $(\mathrm{p}=0.041) . \mathrm{GA}=$ gait aid group, NGA = no gait aid group, $\mathrm{P}=$ paretic and $\mathrm{NP}=$ nonparetic. Error bars are standard deviations (SD). 
Figure 3
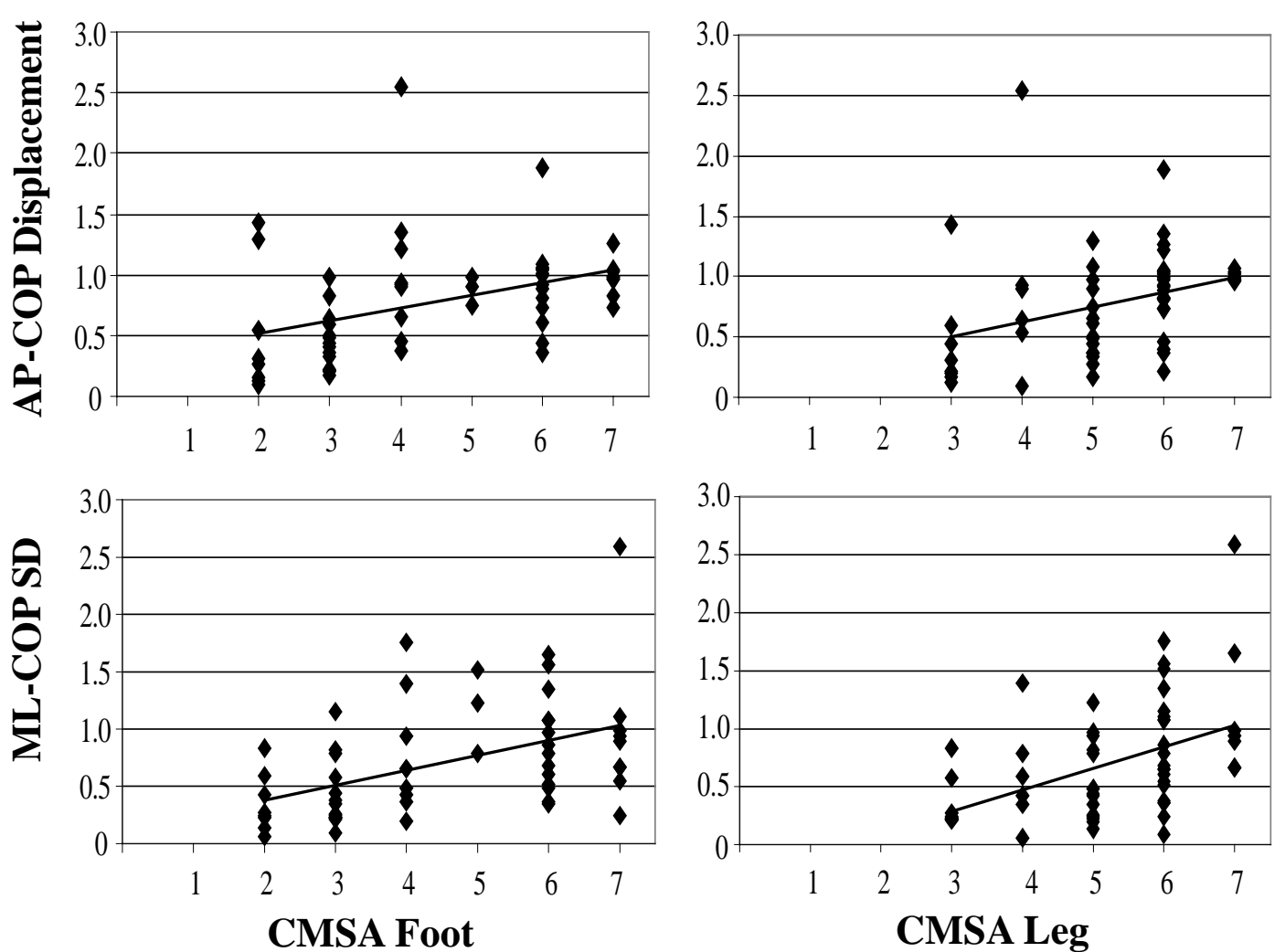

Figure 3: Positive correlations $(\mathrm{DF}=55)$ are illustrated between AP-COP displacement and ML-COP SD ratios (paretic/non-paretic) at preferred speed and CMSA foot ( $\mathrm{r}=0.521$, $\mathrm{p}<0.001 ; \mathrm{r}=0.529, \mathrm{p}<0.001)$ and $\operatorname{leg}(\mathrm{r}=0.485, \mathrm{p}=0.001 ; \mathrm{r}=0.51, \mathrm{p}<0.001)$ scores. 